ARTÍCULO DE INVESTIGACIÓN

\title{
Asociaciones vegetales de los bosques del Municipio de Guanaceví, Durango
}

\author{
Abel García-Arévalo' \\ José Juan Mendoza-Contreras² \\ Jorge Nocedal $^{1}$
}

\begin{abstract}
RESUMEN
Se registran cuatro tipos de vegetación, representados por 10 comunidades vegetales para el Municipio de Guanaceví, Durango. Se reconocen cinco asociaciones vegetales para los bosques de coníferas, 10 para bosques de Pinus-Quercus, nueve para bosques de Quercus-Pinus, cinco para bosques de Quercus y cuatro para el matorral de Arctostaphylos-Quercus. Se describen brevemente las comunidades vegetales que no fueron muestreadas como pastizal, vegetación riparia, vegetación acuática y semiacuática y vegetación propia de sitios de disturbio y áreas agrícolas
\end{abstract}

PALABRAS CLAVE:

Tipos de vegetación, asociaciones vegetales, Guanaceví, Durango.

\begin{abstract}
Records of four vegetation types represented by 10 vegetation communities are presented for the Municipio of Guanaceví, Durango. For mixed-conifer forests five associations were determined, ten for pine-oak forest (Pinus-Quercus), nine for oak-pine forest (Quercus-Pinus), five for oak woodlands (Quercus) and four for manzanita-oak schrub (Arctostaphylos-Quercus). Brief descriptions for those vegetation communities not sampled such as grasslands, riparian vegetation, aquatic and semi-aquatic vegetation, and vegetation characteristic of disturbance and crop lands are also presented.
\end{abstract}

KEY WORDS:

Vegetation communities, plant associations, Guanaceví, Durango. 


\section{INTRODUCCIÓN}

La Sierra Madre Occidental, en lo que corresponde al Municipio de Guanaceví, Durango, es rica en recursos forestales. Esta región sirvió como asentamiento de varios grupos étnicos, entre los que destacan los Tepehuanos, quienes se distinguieron por sus prácticas de cazadores, recolectores y agricultores. La actividad más importante, posterior a la llegada de los españoles, fue la minería, en esos tiempos considerada como la más productiva, destacando varias minas por sus aportaciones de minerales de alta calidad. Otra actividad, a menor escala, fue la agricultura practicada principalmente en las regiones de los valles. La explotación forestal que se realizaba era muy rudimentaria y se restringía a las necesidades de los asentamientos humanos y actividades mineras propias de la región. Posteriormente, los movimientos revolucionarios produjeron la paralización de los trabajos mineros, teniendo entonces que dedicarse a otras actividades productivas, entre ellas la explotación tecnificada de sus bosques.

De los primeros trabajos realizados sobre los bosques de Durango, destaca el de Maysilles (1959), en el cual menciona que en estos bosques se presentan varias especies de Pinus y también de Abies, Pseudotsuga, Cupressus y Juniperus y que estas coníferas están confinadas a las partes altas de la Sierra Madre Occidental, mayormente en altitudes de 2500 m o más. Así mismo, Madrigal (1977) y González (1984), definen y describen los tipos de vegetación del estado. García y González (1998), mencionan las especies de Pináceas que se registran en el estado y su distribución ecológica y por municipios. Gordon (1968), realiza en el sur de Durango un estudio sobre la ecología de Picea chihuahuana, describiendo en forma general la población y el sitio de estudio; concluye que dicho taxón se encuentra en serios problemas de supervivencia. Merlín (1988) en estudios sobre Populus tremuloides (alamillo), en la Sierra de Durango, hace referencia a las poblaciones de esta especie en el Ejido Chiqueros, Municipio de Guanaceví, donde analiza sus principales atributos y algunos aspectos de su ecología.

\section{OBJETIVO}

Determinar los tipos de vegetación y las principales asociaciones vegetales que ocurren en los bosques del Municipio de Guanaceví, Durango.

\section{DESCRIPCIÓN DEL ÁREA DE ESTUDIO}

El Municipio de Guanaceví se localiza al noroeste del Estado de Durango, entre los $25^{\circ} 29^{\prime} 53,06^{\prime \prime}$ y $26^{\circ} 34^{\prime} 23,42^{\prime \prime}$ Norte y $106^{\circ} 36^{\prime} 23,32^{\prime \prime}$ y $105^{\circ} 23^{\prime} 52,81^{\prime \prime}$ Oeste, a una altitud que oscila entre los $2100 \mathrm{~m}$ y $3300 \mathrm{~m}$. Ocupa una superficie de aproximadamente 577000 ha (Fig. 1). Colinda al norte con los municipios de Ocampo y San Bernardo; al oeste con el Estado de Chihuahua; al este con el municipio de El Oro y al sur con el Municipio de Tepehuanes. Fisiográficamente, gran parte del territorio se localiza en la subprovincia Gran Meseta y Cañones Chihuahuenses y una proporción más pequeña, el sur del municipio, es parte de la subprovincia Gran Meseta y Cañones Duranguenses.

Aproximadamente un $80 \%$ del territorio del Municipio de Guanaceví está conformado por rocas sedimentarias y volcanosedimentarias del Paleozoico Inferior de acuerdo con las cartas geológicas INEGI (1991; 1992; 1999a; 1999b).

Para el municipio se registran los siguientes suelos (SPP, 1981): Litosol, que representa aproximadamente un 
$50 \%$ de la superficie total, se localiza en las regiones norte, noreste, este, centro y sur; Regosol, que ocupa cerca del $30 \%$ de la superficie del municipio, se localiza en el occidente, centro-oeste, noroeste y suroeste. Representando superficies más pequeñas, el Cambisol se distribuye en una pequeña porción del sur y noroeste; el Castañozem en porciones del centro y sur; el Feozem en superficies aisladas de las porciones suroeste, sureste y oeste y el Solonetz que se registra para regiones que se ubican en valles, específicamente en la zona sureste.

El Municipio de Guanaceví se localiza en la cuenca hidrológica denominada Nazas-Aguanaval, que constituye una amplia zona cerrada localizada en la Mesa del Norte del país y corresponde a las cuencas endorreicas de los ríos
Nazas y Aguanaval, en los estados de Durango, Coahuila y Zacatecas.

Los climas que se registran para el municipio son los templados subhúmedos con lluvias en verano en más del $60 \%$ de la superficie y el semifrío con verano fresco largo, en la porción occidental de la entidad (García, 1973). Se registran temperaturas de $32{ }^{\circ} \mathrm{C}$ como máxima y $5{ }^{\circ} \mathrm{C}$ como mínima, con una media anual de $23^{\circ} \mathrm{C}$. La precipitación media anual es de $550 \mathrm{~mm}$.

Desde un punto de vista muy general y siguiendo la nomenclatura de Rzedowski (1978), se puede considerar que en los bosques templados de la región predominan las asociaciones vegetales compuestas principalmente por pinos y encinos.

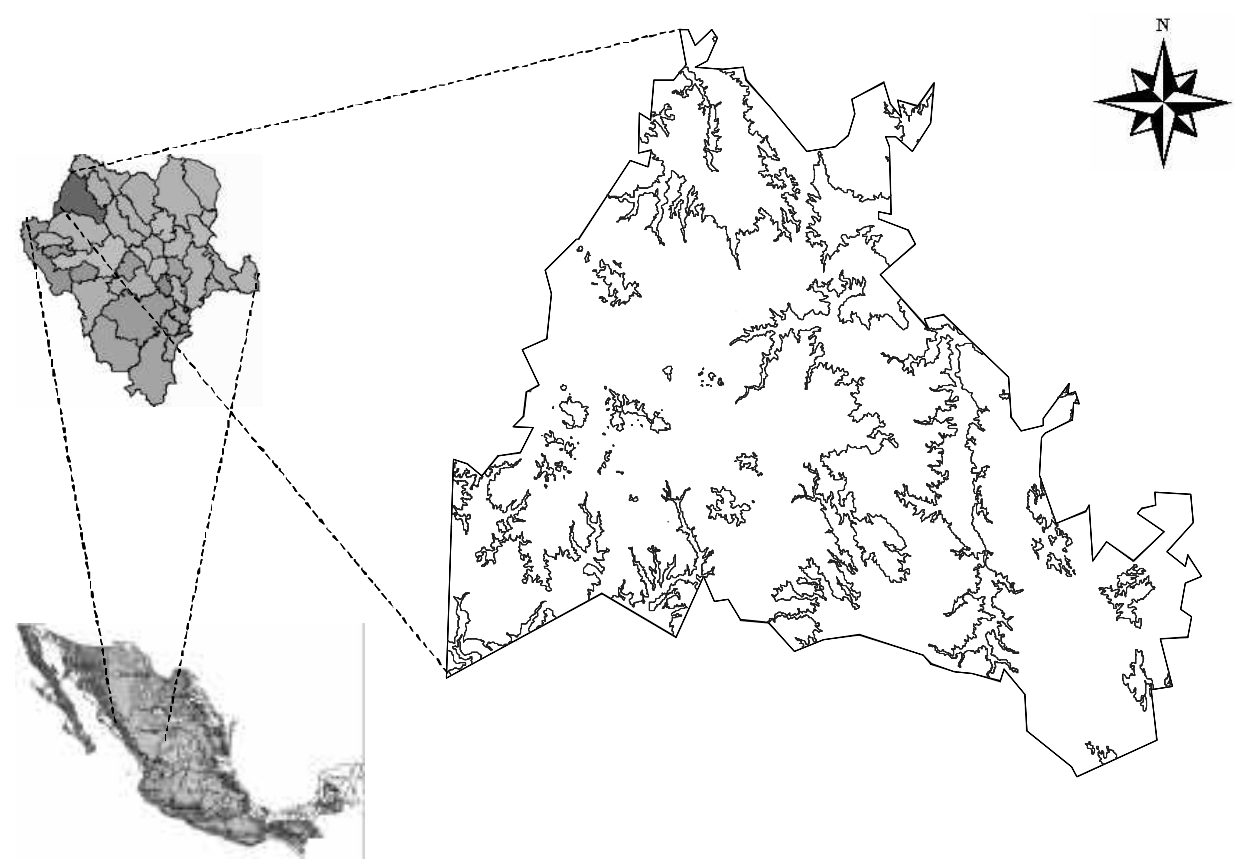

Figura 1. Localización geográfica del Municipio de Guanaceví, Durango. 


\section{METODOLOGÍA}

Para el análisis de la vegetación y la determinación de las principales asociaciones vegetales, se realizaron 71 transectos de longitud variable, utilizando el método de muestreo conocido como cuadrantes centrados en un punto (Mueller-Dombois y Ellenberg, 1974; Brower y Zar, 1977). La longitud del transecto estuvo determinada por la homogeneidad de la vegetación, variando el número de estaciones de cinco a diez, esto es, de $250 \mathrm{~m}$ a $500 \mathrm{~m}$ de longitud. Una vez determinada aleatoriamente la dirección del transecto, cada 50 metros se realizó un muestreo obteniendo cuatro lecturas. En cada cuadrante se midió la distancia a la especie leñosa más cercana y se anotó si estaba viva, muerta o si representaba un tocón o un árbol derrumbado. También se midió el diámetro a la altura del pecho (DAP) y se estimó la cobertura de la copa y la altura del individuo; en el caso de tocones se obtuvo el diámetro basal. Como información ecológica complementaria se registraron coordenadas, altitud, exposición, pendiente, indicios de incendios y ausencia o presencia de plagas y enfermedades. Para estimar la densidad, frecuencia y cobertura de cada especie se utilizaron las ecuaciones convencionales descritas por Brower y Zar (1977). Para determinar los tipos de vegetación y las asociaciones vegetales se utilizaron las tablas de frecuencias y las unidades de muestreo (transectos) se agruparon en clases homogéneas basadas en las especies dominantes.

\section{RESULTADOS}

Se registraron cuatro tipos de vegetación y diez comunidades vegetales para el área boscosa del Municipio de Guanaceví, clasificación que concuerda con las propuestas por Rzedowski (1978) para la República Mexicana y por
González (1984) para el Estado de Durango, las cuales consideran los elementos florísticos y fisonómicos dominantes para la determinación de los tipos.

Los tipos de vegetación representativos de la zona son: Bosque de coníferas, Bosque de Quercus, Pastizal y Vegetación acuática y semiacuática. Siguiendo un gradiente altitudinal se describen las siguientes comunidades: 1 . bosque de Pinus; 2. bosque de Pseudotsuga; 3. bosque de Pinus-Quercus; 4. bosque de Quercus-Pinus; 5. bosque de Quercus; 5a. bosque abierto de Quercus; 6. matorral de Arctostaphylos-Quercus; 7. pastizal; 8. vegetación riparia; 9 . vegetación acuática y subacuática y 10 . vegetación propia de sitios de disturbio y áreas agrícolas.

Cabe mencionar que las comunidades de pastizal, vegetación riparia, vegetación acuática y subacuática y vegetación de sitios de disturbio y áreas agrícolas no fueron muestreados, considerando que el interés radicó en los bosques maderables de importancia económica y zonas de transición, como lo son los bosques abiertos de encino y matorrales de Arctostaphylos. Sin embargo, las comunidades no muestreadas son descritas brevemente.

\section{Bosque de Pinus}

Representan comunidades dominadas por poblaciones monoespecíficas o combinaciones de especies del género Pinus; son más frecuentes en los sitios más altos de la sierra 0 , en el caso particular de Pinus cembroides, en laderas bajas de la vertiente oriental de las sierras.

Bosque de Pinus arizonica. Son bosques monoespecíficos que se distribuyen en las zonas altas de la sierra en altitudes que oscilan entre los $2600 \mathrm{~m}$ y 
los $3000 \mathrm{~m}$ principalmente en mesetas, laderas y cordones montañosos. Se desarrollan en sitios con suelos de mediana a baja calidad; la madera es muy cotizada por lo que estos bosques tienen un alto aprovechamiento maderable en la región.

Bosque de Pinus cooperi. Bosques monoespecíficos que se distribuyen en las zonas más altas del sistema montañoso. Las poblaciones de $P$. cooperi se presentan en un gradiente altitudinal que va de los $2500 \mathrm{~m}$ a los $2800 \mathrm{~m}$. Se caracteriza por presentar solamente un estrato arbóreo por árboles de hasta $25 \mathrm{~m}$ de altura, dichas poblaciones se ubican principalmente en zonas denominadas "bajíos", con suelos relativamente profundos y presencia de materia orgánica. Florísticamente, son comunidades con una gran diversidad de especies, destacando elementos de las familias de las compuestas, gramíneas, liliáceas y leguminosas, entre otras. A nivel de aprovechamientos forestales, son los bosques preferidos para la extracción, por presentar las mejores características de altura y diámetro de los árboles; por otra parte, la madera de Pinus cooperi, es una de las más solicitadas en el mercado regional.

Bosque de Pinus arizonica-P. cooperi. Se localizan principalmente en las partes altas de ambas vertientes de la sierra y frecuentemente bajan hasta la cota altitudinal de los $2500 \mathrm{~m}$, a través del origen de cañadas que dirigen los arroyos temporales hacia las partes más bajas. Se caracteriza por un estrato arbóreo establecido por la asociación de Pinus arizonica-P. cooperi, con árboles de hasta $20 \mathrm{~m}$ de altura. Esporádicamente puede presentarse un estrato arbustivo con algunas especies de Quercus spp., Arbutus glandulosa, Juniperus deppeana y Arctostaphylos pungens. Se desarrollan en sitios con suelos poco profundos con drenaje deficiente, en suelos pedregosos y con afloramientos rocosos. Son abundantes especies de las familias de las compuestas, liliáceas y gramíneas. Se registraron aprovechamientos forestales en estas comunidades.

\section{Bosque de Pinus chihuahuana-Pinus} engelmannii. Las comunidades de este tipo están poco representadas en el área; se localizan en laderas secas de exposición oriental o sitios denominados localmente "puertos" con amplia exposición a la luz solar y se distribuyen entre los 2 $400 \mathrm{~m}$ y $2620 \mathrm{~m}$. Los bosques son abiertos y frecuentemente presentan un estrato arbustivo abierto o denso dominado por Quercus depressipes, Arctostaphylos pungens y Arbutus arizonica; en el estrato herbáceo destacan algunas especies de los géneros Senecio, Salvia, Viguiera, Commelina, Milla, Tagetes, Trifolium, Conyza y Lopezia, entre otras. En general, estos bosques se presentan en suelos de color blanco de substrato pobre con fuerte afloramiento rocoso.

Bosque de Pinus cembroides. Son bosques que se distribuyen en laderas bajas de ambas vertientes de las sierras del norte, este y sureste del municipio; corresponden a la franja de vegetación intermedia entre el pastizal y matorrales bajos que delimitan la presencia de los bosques de mayor porte, encinos y pinos que se desarrollan en mayores altitudes. Presentan una variación altitudinal que va de los $2150 \mathrm{~m}$ a los $2350 \mathrm{~m}$, en laderas medianamente abruptas, suaves y expuestas, con suelos someros pedregosos y áreas de fuerte afloramiento rocoso. Se distinguen por la presencia de elementos de $2 \mathrm{~m}$ a $5 \mathrm{~m}$ de altura y frecuentemente son bosques monoespecíficos abiertos. Ocasionalmente hay presencia de otros elementos arbustivos, como es el caso de algunas especies del género Opuntia, Quercus, Acacia schaffneri, Juniperus erythrocarpa, entre otros, considerando la transición hacia partes bajas o altas. La característica de 
presentar amplios claros en la comunidad, permite la presencia de una alta diversidad de especies herbáceas, son comunes Senecio albolutescens, Stevia serrata, Viola barroeteana, Gomphrena decumbens, Tagetes microphylla, Salvia laevis, Erodium cicutarium y Lithospermum cobrense.

\section{Bosque de Pseudotsuga}

Representa bosques muy particulares con requerimientos topográficos y de humedad muy especiales; la calidad de sitio de estos bosques en general es muy buena. Se desarrollan en cañones, laderas húmedas y protegidas y sitios expuestos a humedad ambiental y cauces de arroyos, en las partes altas, en altitudes entre los $2400 \mathrm{~m}$ y $3000 \mathrm{~m}$. Frecuentemente se asocian a Pseudotsuga menziesii otras especies con requerimientos ecológicos similares, como es el caso de Pinus arizonica, Pinus ayacahuite, Cupressus Iusitanica, Populus tremuloides, Picea chihuahuana, Quercus sideroxyla y Juniperus deppeana, entre otras (Fig. 2).

\section{Bosque de Pinus-Quercus}

Estos bosques mixtos son comunidades en donde los pinos predominan y son acompañados, en una proporción más baja, por encinos; son característicos de las zonas altas de las montañas, ubicándose en diversas exposiciones y con diferentes composiciones florísticas; generalmente presentan una alta diversidad de especies vegetales.

Bosque de Pinus arizonica-Quercus sideroxyla. Son comunidades vegetales que se distribuyen a menores altitudes que los bosques de $P$. arizonica; en terrenos con pendientes suaves $y$ abruptas y altitudes de $2500 \mathrm{~m}$ a 2700 $\mathrm{m}$. Los suelos presentan una capa importante de materia orgánica $y$, generalmente, son sitios muy productivos que contienen una importante dotación de humedad ambiental.

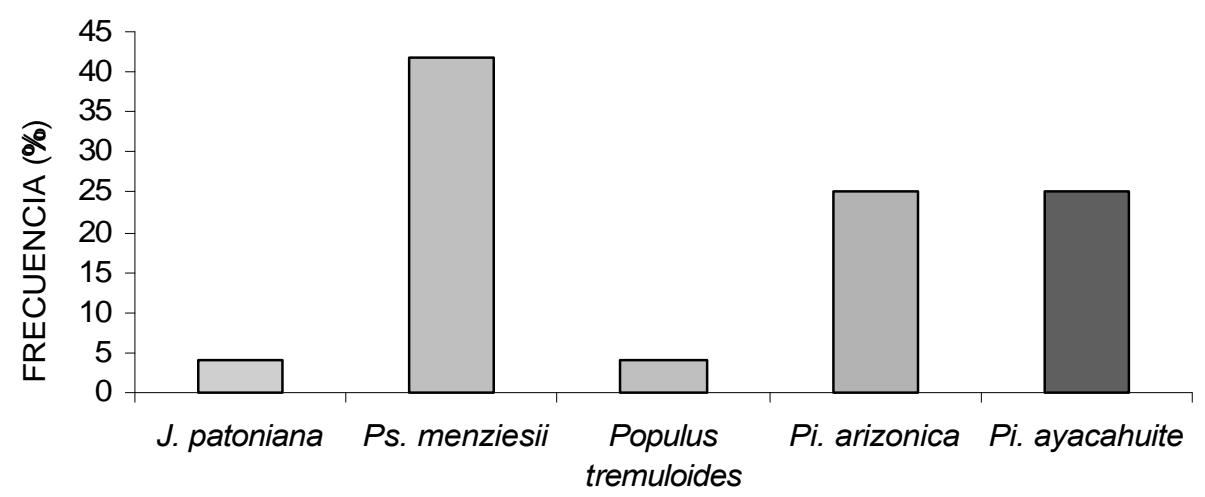

ESPECIES

Figura 2. Frecuencia de ocurrencia de las especies arbóreas y arbustivas el bosque de Pseudotsuga menziesii. 
Bosque de Pinus cooperi-Quercus sideroxyla. Son comunidades vegetales que se distribuyen en terrenos con pendientes suaves y abruptas y con altitudes de 2500 m a $2700 \mathrm{~m}$; los suelos presentan una capa importante de materia orgánica. En los sitios con pendientes suaves generalmente se desarrollan bosques abiertos y en sitios con mayor pendiente es frecuente encontrar comunidades más densas; los principales componentes son, Pinus cooperi y Quercus sideroxyla en el estrato arbóreo (Fig. 3) y puede o no existir un estrato arbustivo en el cual destacan Arctostaphylos pungens, Quercus depressipes y Quercus striatula.

Bosque de Pinus teocote-Quercus rugosa. Corresponde a bosques que se distribuyen principalmente en cañadas con pendientes abruptas en altitudes que van de los $2500 \mathrm{~m}$ a los $2700 \mathrm{~m}$; los suelos son ricos en materia orgánica, por las altas concentraciones de hojarasca. Los elementos dominantes son Pinus teocote y Quercus rugosa; frecuentemente pueden presentarse otros elementos arbóreos en una proporción más baja, como es el caso de Quercus crassifolia, Pinus durangensis, $P$. lumholtzii y Arbutus glandulosa. En el estrato arbustivo suelen presentarse Arctostaphylos pungens, Quercus depressipes y Juniperus durangensis.

\section{Bosque de Pinus cembroides- Quercus oblongifolia y/o Quercus chihuahuensis. Estas comunidades vegetales se caracterizan por presentar un estrato arbóreo dominado por Pinus cembroides, Quercus oblongifolia y/o $Q$. chihuahuensis, con árboles de hasta $8 \mathrm{~m}$ de altura, en altitudes que oscilan entre los $2200 \mathrm{~m}$ y los $2450 \mathrm{~m}$ en cañadas y fondos de cañadas.}

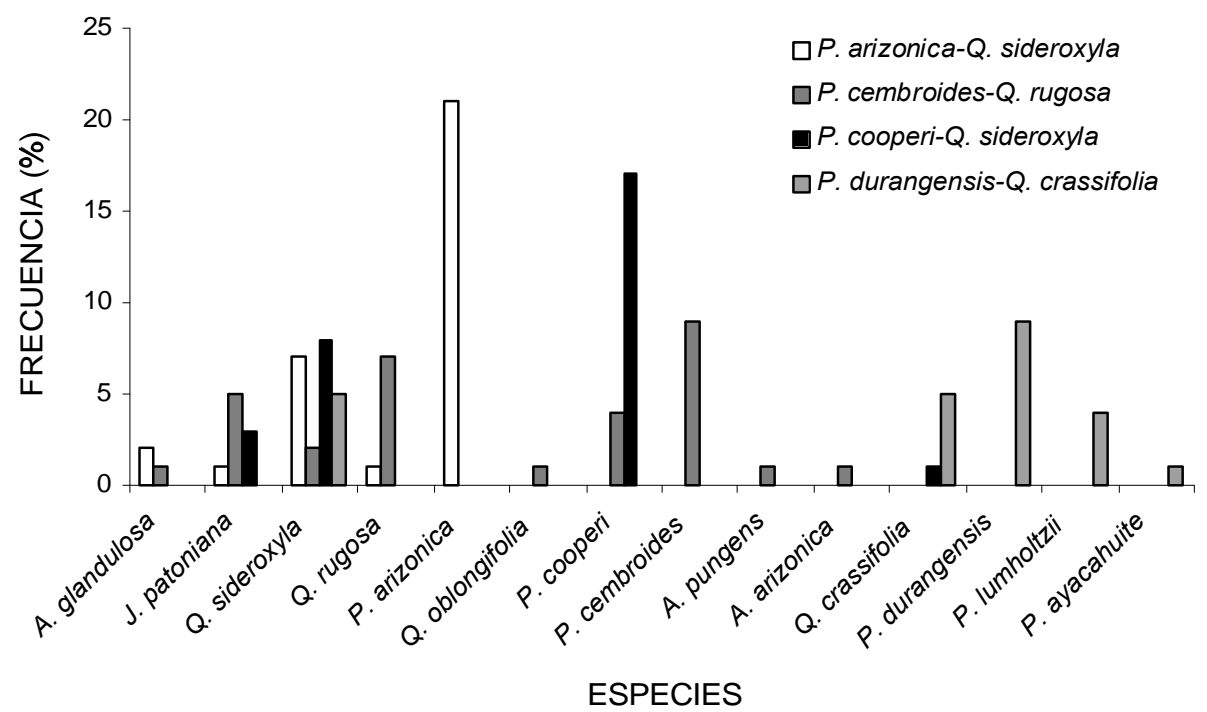

Figura 3. Frecuencia de ocurrencia de las especies arbóreas y arbustivas en cuatro asociaciones de Pinus-Quercus en el Municipio de Guanaceví, Durango. 
Bosque de Pinus cembroides-Quercus emoryi. Representa asociaciones vegetales en donde predominan Pinus cembroides y Quercus emoryi; se desarrollan en las estribaciones de la sierra, justo en el límite donde inician las comunidades vegetales de pastizales y matorrales; estos bosques se presentan estructurados en forma semidensa, abierta y en ocasiones densa, principalmente en cañadas cerradas. Los elementos acompañantes pueden ser de ambientes más templados como es el caso de $P$. chihuahuana, $P$. engelmannii, Quercus rugosa, entre otros, o en arroyos temporales con elementos riparios como Cupressus, Fraxinus, Salix, Populus y Carya, o bien de ambientes más secos y cálidos como Juniperus, Mimosa, Acacia y Opuntia spp.

Otras asociaciones de pino-encino que se registraron en menor cantidad en el municipio son los bosques de Pinus durangensis-Quercus crassifolia, $P$. cembroides- $Q$. rugosa, $P$. durangensis- $Q$. fulva, $P$. lumholtzii-Q. crassifolia y $P$. leiophylla-Q. crassifolia.

\section{Bosque de Quercus-Pinus}

Son bosques mixtos que se distribuyen desde las partes altas de montaña hasta las zonas de transición con las comunidades de pastizales y matorrales, predominan las especies del género Quercus sobre las de Pinus. Conforman bosques diversos, desde muy densos en las zonas altas a muy abiertos como los bosques de Quercus emoryi-Pinus cembroides. Presentan un alto nivel de diversidad de especies vegetales por su amplio rango de distribución.

\section{Bosque de Quercus sideroxyla-Pinus} arizonica. Los bosques mixtos de estas especies se desarrollan en altitudes de $2650 \mathrm{~m}$ a $2800 \mathrm{~m}$ y representan comunidades densas en exposiciones que presentan mayor influencia de humedad; generalmente están constituidos de tres estratos, el arbóreo representado por estas dos especies en términos de dominancia y en forma aislada pueden estar presentes Pinus teocote y Quercus rugosa; en el estrato arbustivo son característicos Arbutus glandulosa, Juniperus deppeana, Ceanothus buxifolius y Arctostaphylos pungens. El estrato herbáceo presenta una alta diversidad, siendo más representativas las familias de las compuestas, las gramíneas, las liliaceas y las leguminosas.

\section{Bosque de Quercus sideroxyla-Pinus} cooperi. Se distribuye en altitudes de los $2500 \mathrm{~m}$ a los $2600 \mathrm{~m}$ y forman bosques semidensos a densos, el substrato generalmente es rico en nutrientes por la alta concentración de materia orgánica. Esporádicamente pueden presentarse otros elementos arbóreos como Arbutus glandulosa, Quercus rugosa, Pinus leiophylla, Quercus crassifolia; en el estrato arbustivo son frecuentes Arbutus arizonica y Arctostaphylos pungens (Fig. 4).

Bosque de Quercus chihuahuensisPinus cembroides. Son bosques que se desarrollan en altitudes de $2300 \mathrm{~m}$ a $2580 \mathrm{~m}$ y se distribuyen ampliamente en laderas expuestas, fondos de cañadas o en espacios abiertos a semiabiertos; se distinguen por la dominancia en el estrato arbóreo de Quercus chihuahuensis y Pinus cembroides, también pueden presentarse elementos aislados de Pinus chihuahuana, P. engelmannii, P. cooperi, Quercus rugosa y Juniperus deppeana.

\section{Bosque de Quercus emoryi-Pinus} cembroides. Representa asociaciones vegetales sencillas en cuanto a su estructura, con dominancia de Quercus emoryi y Pinus cembroides; frecuentemente pueden presentarse otros elementos, tanto de ambientes templados como xerófilos. Estos bosques se distribuyen princi- 


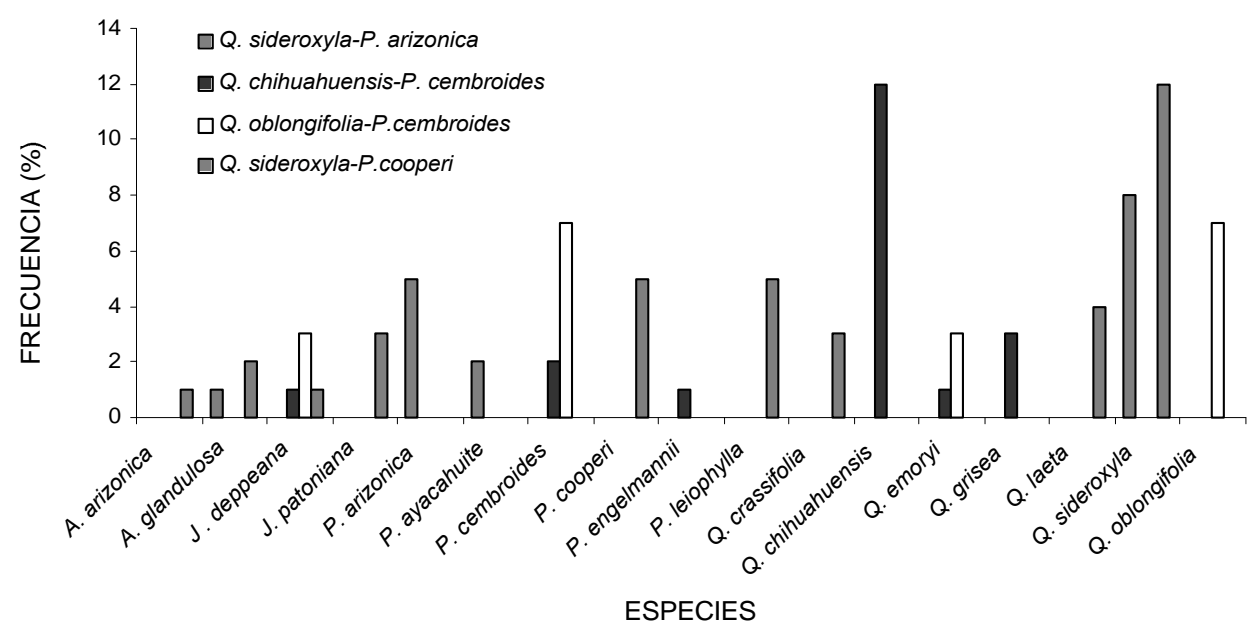

Figura 4. Frecuencia de ocurrencia de las especies arbóreas y arbustivas en cuatro asociaciones de Quercus-Pinus en el Municipio de Guanaceví, Durango.

palmente en exposiciones de laderas secas con suelos someros y con afloramientos rocosos en altitudes de $2100 \mathrm{~m}$ a $2550 \mathrm{~m}$.

También se registraron, pero en menor ocurrencia, las asociaciones de Quercus emoryi-Pinus chihuahuana, Q. oblongifolia-P. cembroides, $Q$. sideroxyla$P$. durangensis, $Q$. sideroxyla-P. ayacahuite y $Q$. sideroxyla-P. leiophylla.

\section{Bosque de Quercus}

Dentro de estas comunidades quedan incluidos los bosques monoespecíficos o asociaciones de especies del género Quercus; se distribuyen en diversos ambientes desde altitudes de 2 $200 \mathrm{~m}$ en laderas secas y en la ecotonía con los pastizales, hasta los $2500 \mathrm{~m}$ en cañadas protegidas y a lo largo de arroyos temporales en las estribaciones de la sierra.
Bosque de Quercus chihuahuensis. Las comunidades vegetales dominadas por esta especie; generalmente se distribuyen en laderas de pendientes suaves en lomeríos y abruptas en cañadas en altitudes de $2200 \mathrm{~m}$ a $2450 \mathrm{~m}$. Otros elementos que forman parte de estas unidades de paisaje en una proporción baja, son Juniperus erythrocarpa, Acacia schaffneri, Pinus cembroides, $P$. chihuahuana, $P$. engelmannii, Arbutus arizonica, Opuntia spp. y Agave parryi, principalmente.

Bosque de Quercus oblongifolia. Se distribuyen principalmente en cañadas protegidas de pendientes suaves, siguiendo el curso de arroyos temporales, en altitudes de $2100 \mathrm{~m}$ a $2300 \mathrm{~m}$. En el estrato arbustivo cuando se presenta, es común observar a Mimosa biuncifera, Acacia schaffneri y Opuntia spp., entre otras.

Bosque de Quercus emoryi. Estas comunidades se caracterizan por 
presentar una estructura arbórea sencilla, desarrollarse en laderas de pendientes suaves y generalmente constituyen bosques abiertos con amplios claros en el sotobosque, fomentando la presencia de un estrato herbáceo con una riqueza específica relativamente alta; se presentan en altitudes de $2100 \mathrm{~m}$ a 2400 $\mathrm{m}$ y se desarrollan en sitios de suelos frecuentemente pobres con afloramientos rocosos. Se pueden asociar especies arbustivas principalmente de ambientes xerófilos.

\section{Bosque de Quercus emoryi-Quercus oblongifolia. Esta asociación vegetal es común en zonas de cañadas abiertas entre los 2100 m y 2300 m, general- mente asociada a cauces de arroyos temporales, con suelos pedregosos y con cierta sedimentación del material acarreado de las partes altas. De manera frecuente se pueden encontrar otros elementos asociados como es el caso de Pinus cembroides, Acacia schaffneri, Mimosa biuncifera, Juniperus spp. y Fouqueria splendens, entre otras.}

Bosque de Quercus chihuahuensisQuercus emoryi. Es una asociación vegetal que se distribuye entre los 2200 $\mathrm{m}$ y $2300 \mathrm{~m}$, en pendientes suaves y suelos compactos con afloramientos rocosos, Quercus chihuahuensis y $Q$. emoryi son los elementos dominantes y es común en este tipo de comunidades la ausencia de otras especies leñosas. Puede ocurrir una alternancia dependiendo del hábitat, en la dominancia de dichos elementos tornándose un bosque de Quercus chihuahuensis-Q. emoryi a un bosque de Quercus emoryi-Q. chihuahuensis, según sea el caso.

\section{5a. Bosque abierto de Quercus spp.}

Son bosques de encino con las mismas características y elementos florísticos dominantes descritos anteriormente para los bosques de Quercus. Se distinguen por presentar una distribución más dispersa de sus elementos creando bosques más abiertos que dan otra fisonomía al paisaje.

\section{Matorral de Arctostaphylos-Quercus spp.}

Son comunidades vegetales que se distinguen por presentar como dominantes fisonómicos a Arctostaphylos pungens (manzanita) en combinación con especies del género Quercus (encino) formando matorrales densos, en ocasiones impenetrables, que no alcanzan alturas superiores a los $3 \mathrm{~m}$. Generalmente se asocian a sitios impactados, principalmente por incendios $u$ otro tipo de agentes de disturbio. Este tipo de asociación coloniza rápidamente las áreas impactadas, no permitiendo el crecimiento de otro tipo de asociaciones vegetales. Suele presentarse en un gradiente altitudinal muy variable. Las especies de encino más representativas que acompañan a la manzanita en estas comunidades son: Quercus depressipes, Q. striatula, $Q$. chihuahuensis y $Q$. emoryi.

En sitios ya modificados en donde predominaban los bosques mixtos y han sido favorecidas las poblaciones de $A$. pungens, frecuentemente se distinguen aisladas las especies que dominaron fisonómicamente el paisaje, como se representa en la figura 5 con Pinus leiophylla y Quercus sideroxyla.

\section{Otros tipos de Vegetación}

Pastizal. Vegetación constituida por un solo estrato, en donde las especies dominantes son gramíneas perennes y anuales. Se distribuye en las partes bajas y lomeríos bajos del municipio, en sitios generalmente con suelos profundos y 
drenaje deficiente. En algunos casos suelen asociarse con especies semileñosas y leñosas sobre todo en áreas con indicios de perturbación. En general, una buena extensión de pastizales dentro del municipio se pueden considerar como inducidos, atribuyendo esto al aprovechamiento intensivo y extensivo que se ha dado durante años a través de la ganadería y a la apertura de nuevas zonas de cultivo.

Las especies más representativas de este tipo de vegetación son: Bouteloua gracilis, B. hirsuta, B. barbata, Aristida orcuttiana, A. schiedeana, así como Bromus spp., Chloris spp., Muhlenbergia spp., Sporobolus spp. y Panicum spp., entre otras. En forma aislada pueden asociarse especies arbustivas como Acacia schaffneri, Mimosa biuncifera, Opuntia spp. y especies herbáceas princi- palmente de las familias de las compuestas, leguminosas, liliáceas y ciperáceas.

Vegetación riparia. Es el tipo de vegetación leñosa que se asocia a corrientes de agua que desde las partes altas de la sierra, se conduce hacia las partes más bajas de la cuenca; comparada con con otros tipos de vegetación, es una de las menos representadas en la región. A las mayores altitudes, son comunes especies de los géneros Pinus, Pseudotsuga, Populus y Quercus y en localidades más bajas (2 200 m a 2400 m), son comunes Populus, Salix, Carya, Cupressus, Fraxinus y, de hábitos trepadores se distinguen Lonicera pilosa y Vitis berlandieri. En la región, este tipo de vegatación es más notoria en el cauce del Río Sextín-Nazas y sus afluentes.

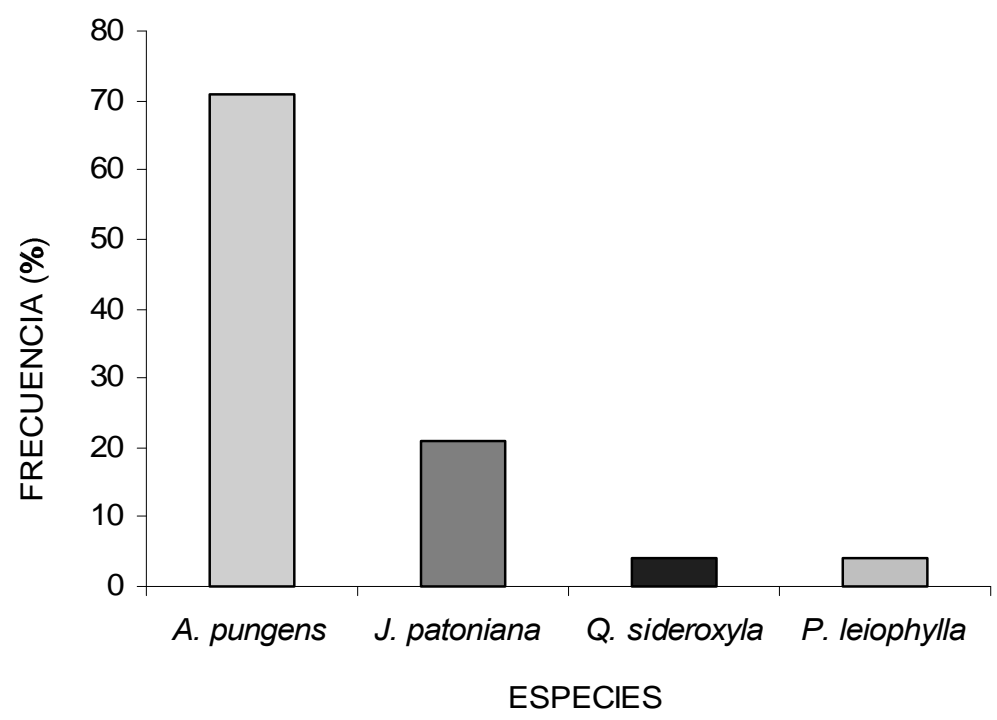

Figura 5. Frecuencia de ocurrencia de las especies arbóreas y en el matorral de Arctostaphylos pungens. 
Vegetación acuática y semiacuática. Es el tipo de vegetación que se encuentra ligada a cuerpos de agua, ríos, arroyos permanentes, sitios inundables, ciénagas y ojos de agua. Es uno de los tipos de vegetación de menor representación en la región y a futuro podría reducirse de manera alarmante, por la disminución o ausencia del agua. En términos de conservación, se considera el ecosistema más frágil y vulnerable. Los géneros de ciperáceas Carex y Cyperus son muy abundantes y forman colonias densas y conspicuas. De las acuáticas sumergidas y flotantes se registran Potamogeton nodosus y Nymphoides fallax; en sitios propensos a inundaciones y con drenaje deficiente son comunes algunas especies de los géneros Carex, Cyperus, Echinochloa, Sporobolus, Allium, Mimulus y Eryngium, entre otras.

\section{Vegetación de sitios de disturbio y de} áreas agrícolas. Este tipo es resultante del efecto de las principales actividades antropogénicas, corresponde a la representativa de las orillas de caminos, asociada a cultivos abandonados 0 funcionales y asentamientos humanos, donde el hábitat natural ha sido modificado. Las especies más conspicuas en este tipo de vegetación, a orillas de caminos y carreteras, son: Brickellia vernicosa, Solanum eleagnifolium, Andropogon villosum, Tithonia tubaeformis, Sphaeralcea angustifolia, Lepidium virginicum, Mimosa biuncifera y Nicotiana glauca. En terrenos de cultivo abandonados o no utilizados en la temporada, una especie común en primavera es Argemone ochroleuca y, en época de lluvias, se distinguen principalmente especies de compuestas, gramíneas y leguminosas.

\section{DISCUSIÓN Y CONCLUSIONES}

Los tipos y unidades de vegetación registrados para el Municipio de
Guanaceví, Durango, en general, presentan amplia distribución en el estado, con mayor representatividad en las regiones montañosas; sin embargo, vale la pena recalcar que algunas de las asociaciones descritas, representan comunidades muy especiales desde el punto de vista ecológico, entre ellas podemos destacar los bosques de Pseudotsuga y la vegetación riparia que incluyen componentes de distribución muy restringida.

Los bosques monoespecíficos de Pinus cooperi son de suma importancia desde el punto de vista comercial y mantienen una continuidad en la entidad en su distribución con la mayor parte de la geografía del estado. Así mismo, los bosques de Pinus arizonica de distribución más norteña y a mayores altitudes sobresalen por su alto valor ecológico y comercial. Ambos bosques monoespecíficos se distinguen por presentar una gran presión y sus poblaciones se han visto afectadas en cantidad y calidad en la región.

La vegetación acuática y semiacuática, por su dependencia directa del agua, representa un tipo de vegetación muy frágil, considerando la disminución gradual y constante de los cuerpos y corrientes de agua.

Los bosques mixtos son los de mayor distribución en el municipio y, al igual que los bosques de Pinus cooperi y $P$. arizonica, presentan evidencia importante de deterioro, por contener elementos maderables de valor comercial.

Las actividades productivas, la falta de un manejo sustentable de los recursos y el tiempo e intensidad con que estos han sido aprovechados, se identificaron como los principales motivos de la modificación de los ecosistemas naturales, provocando que especies de interés sean 
desplazadas y que los nichos que dejan sean utilizados por especies de menor valor comercial y de mayor plasticidad genética, que se tornan muy evidentes con el aumento de los matorrales de Arctostaphylos y Quercus spp. en las zonas forestales.

La vegetación asociada a los asentamientos humanos y áreas agrícolas gradualmente ha ido en aumento y en los últimos años se ha visto favorecido el crecimiento de la frontera agrícola y la retracción de la frontera forestal.

\section{RECONOCIMIENTOS}

El presente trabajo se realizó con fondos del Instituto de Ecología A.C. clave 904-61. Agradecemos al M. en C. Efraín Rodríguez el apoyo otorgado en los muestreos de vegetación y al INECOL por el apoyo otorgado durante el trabajo de campo y gabinete. Esta es una adaptación del trabajo de tesis del segundo autor en la Escuela de Ciencias Forestales de la Universidad Juárez del Estado de Durango.

\section{REFERENCIAS}

Brower, J.E. y J.H. Zar. 1977. Field and laboratory methods for general ecology. Wm. C. Brown Company Publishers. Dubuque, lowa. 194 pp.

García A., A. y M.S. González E. 1998. Pináceas de Durango. Primera edición. Durango, Durango. 179 p.

García, E. 1973. Modificaciones al sistema de clasificación climática de Koeppen. Segunda edición. Instituto de Geografía. Universidad Nacional Autónoma de México. México, D.F. $246 \mathrm{p}$.
González E., S. 1984. La vegetación de Durango. Cuadernos de Investigación Tecnológica CIIDIR-IPN Unidad Durango 1(1):1-114.

Gordon A.G. 1968. Ecology of Picea chihuahuana Martínez. Ecology 49:880-896.

INEGI. 1991. Carta geológica, escala 1:250 000. Guachochi G13-4.

INEGI. 1992. Carta geológica, escala 1:250 000. Hidalgo del Parral G13-5.

INEGI. 1999a. Carta geológica, escala 1:250 000. Pericos G13-7.

INEGI. 1999b. Carta geológica, escala 1:250 000. Santiago Papasquiaro G13-8.

Madrigal, X. 1977. Características generales de la vegetación del Estado de Durango. Ciencia Forestal 2(7):30-58.

Maysilles, J.H. 1959. Floral relationships of the pine forests of western Durango, Mexico. Tesis doctoral. Univ. Michigan. Ann Arbor, Michigan. $165 \mathrm{p}$.

Merlín, B.A. 1988. Uso actual y perspectivas del Populus tremuloides Michx. en el Estado de Durango. Tesis de licenciatura. Universidad Autónoma Chapingo. Chapingo. México. 46 p.

Mueller-Dombois, D. y $\mathrm{H}$. Ellenberg. 1974. Aims and methods of vegetation ecology. John Wiley and Sons, Inc. Nueva York. 547 p.

Rzedowski, J. 1978. Vegetación de México. Editorial Limusa. México, D.F. 423 p. 


\section{SPP (Secretaria de Programación y \\ Presupuesto). 1981. Carta edafológica \\ Chihuahua escala 1:1 $000000 . \diamond$}

Manuscrito recibido el 10 de abril de 2003.

Aceptado el 25 de febrero de 2004.

Este documento se debe citar como:

García-Arévalo, A.; J.J. Mendoza-Contreras y J. Nocedal. 2004. Asociaciones vegetales de los bosques del Municipio de Guanaceví, Durango. Madera y Bosques 10(1):21-34. 